\title{
A Tentative Approach to Ambiguity in English Sentences
}

\author{
Weiwei Dai \\ Southwest Minzu University, Chengdu, China \\ Email: 7289209@qq.com
}

How to cite this paper: Dai, W.W. (2021) A Tentative Approach to Ambiguity in English Sentences. Open Access Library Journal, 8: e7918.

https://doi.org/10.4236/oalib.1107918

Received: September 1, 2021

Accepted: September 27, 2021

Published: September 30, 2021

Copyright (c) 2021 by author(s) and Open Access Library Inc.

This work is licensed under the Creative Commons Attribution International License (CC BY 4.0).

http://creativecommons.org/licenses/by/4.0/

\section{(c) (i) Open Access}

\begin{abstract}
As a common language phenomenon, ambiguity exists in various languages, and English is no exception. While ambiguity makes the language lively, and interesting, it also brings great troubles to the learning and practice of English. This article tries to classify English ambiguity from four aspects: phonetics, lexical, syntactical, and pragmatic aspects. It aims to clarify the causes of English language ambiguity. At the same time, it points out that only when sentences are placed in a specific language context can the semantics be clarified. Through the analysis of ambiguous sentences, we hope to deepen the understanding of English language and improve the effectiveness of English language communication.
\end{abstract}

\section{Subject Areas}

Linguistics

\section{Keywords}

Ambiguity, Syntax, English

\section{Introduction}

Language is a social phenomenon established by conventions, not created by people according to scientific laws. There is often no necessary one-to-one correspondence between sentences and semantics. Different words can often express the same meaning; the same words and sentences can also express different meanings. In other words, there are a lot of multi-word or multi-sense words in the language, which is the ambiguity in the language. Two grammars in modern England, Jocobs and Rosendbaum offer us an excellent example of ambiguity sentence in their work English Transformational Grammar. "The Seniors were told to stop demonstrating on campus" [1]. This simple sentence has six differ- 
ent interpretations:

1) The seniors were demonstrating and were asked on campus to desist.

2) The seniors were demonstrating and were asked to desist on campus (although they could demonstrate elsewhere).

3) The seniors were demonstrating on campus and were asked to desist.

4) People were demonstrating on campus and seniors were asked to stop them.

5) People were demonstrating and seniors were asked on campus to stop them.

6) People were demonstrating and seniors were asked to stop them from doing so on campus (although they could do it elsewhere).

Such a single sentence can has 6 different interpretations is due to the ambiguity function of "the seniors" and "on campus". "The seniors" can act as the agent as well as the patient of the action "to stop", while adverbial phrase "on campus" also has different locative roles for the action of "to tell", "to stop" and "to demonstrate".

A word, phrase, or sentence can be used for at least two or more interpretations, which is called "ambiguity" Ambiguity is a characteristic shared by human languages. No matter what kind of natural language is used to communicate, you will feel the confusion from the language itself to varying degrees, such as the semantic vagueness that causes difficulty in understanding, or the ambiguity that has been rhetorically processed to achieve a certain stylistic effect (equivocation and other obscure words). British semantic scholar Ruth M. Kempson pointed out that "words and sentences may have more than one meaning" [2]. Wu Qianguang, a famous Chinese scholar, pointed out in his monograph "Introduction to Semantics": “Ambiguous' refers to a sentence that you can do two or more kinds of explanation" [3].

It can be seen that ambiguity is a complex language phenomenon. It means that an expression is ambiguous and can be interpreted in two or more ways. Yet ambiguity is a common phenomenon in language communities. Phonetics, grammar, semantics, syntax, as small as punctuation and intonation can all be the cause of ambiguity. Based on this, linguists divide ambiguity into different types such as phonetic ambiguity, lexical ambiguity, syntactic ambiguity, and pragmatic ambiguity.

This paper is divided into three parts. Part 1 is a general introduction which briefly introduces why this topic is chosen and how it is going to be dealt. It shows the scope, perspectives and purposes of the research. Part 2 deals the classification of ambiguity in English sentences at different linguistic (phonetic, lexical, pragmatic, syntactic) level. It emphasizes syntactical ambiguity rather than the others. Part 3 is the conclusion of the paper, which points out the importance of proper handling of ambiguity in English sentences.

\section{Classification of Ambiguity in English Sentences}

\subsection{Phonetic Ambiguity}

Phonetic ambiguity is mainly because in people's daily life, voice always carries 
information in the form of language flow as the main transmission medium. Due to the lack of visual aids and necessary context of written language and text, ambiguity in language is unavoidable.

E.g. 1: A classic example of phonetic ambiguity in English is a conversation between a mouse and a girl named Alice from Lewis Carroll's book Alice's Adventures in wonderland [4].

"Mine is a long and a sad tale!" said the Mouse, turning to Alice, and sighing.

"It IS a long tail, certainly," said Alice, looking down with wonder at the Mouse's tail; "but why do you call it sad?"

Thus Alice can understand tail can be long, but she wonders why the tail is sad.

In the book Through the Looking Glass from the same author, there are also other examples of phonetic ambiguity [5]:

E.g. 2: Here the Red Queen began again. "Can you answer useful questions?" she said. "How is bread made?"

"I know that! "Alice cried eagerly. "You take some flour-"

"Where do you pick the flower?" the White Queen asked. "In a garden, or in the hedges?"

"Well, it isn't picked at all," Alice explained: "it's ground-"

"How many acres of ground?" said the White Queen. "You mustn't leave out so many things."

In this conversation, ambiguity is caused by two pairs words, "flower" and "flour", the verb "ground" (p.p. form of the verb grind,) which means to crash sth. to very small pieces, and the noun "ground" (a piece of land). The two words in each pair has no semantic relation at all, however, the same pronunciation connects them together. Thus, a contrast of meanings occurs. The White Queen doesn't mean to be humorous, but she unintentionally asked in a humorous manner.

\subsection{Lexical Ambiguity}

One of the biggest characteristics of natural language is the complex relationship between language form and language meaning. For the needs of scientific research, the relationship between the signifier and the signified of the sign is one-to-one for the various artificial languages designed by people. In natural languages, one signifier often corresponds to multiple referents, resulting in ambiguity in vocabulary.

As a symbol of communication, each word of language must correspond to a certain function or meaning. However, due to the finiteness of English symbols and the infinite nature of things, the language cannot perform its communicative functions smoothly when the symbol is the only one. In this way, polysemy, which means a word has multiple meanings, appears, and has a tendency to expand. This is the result of people constantly giving words new meanings in the long-term development of the language. When two or more different meanings 
of a polysemous word are both valid in the same sentence, resulting in the non-unique referent, ambiguity arises.

E.g. 3: Hugo is drawing a cart.

Here two interpretations can be implied from this sentence because the word "draw" can have two different meanings as the predicate in the sentence. So it can be paraphrased as below:

a. Hugo is drawing a picture of a cart.

b. Hugo is pulling a cart.

E.g. 4: They can fish.

The two meanings of this ambiguous sentence are "they can fish" and "they canned fish." "Can" is a homophone. In the first semantic expression, "can" is an auxiliary verb and "fish" is a verb; in the second semantic expression, "can" is a verb and "fish" is a noun. To avoid ambiguity, modal verbs can be added before "can", or general past tense suffixes can be added at the end of words, and "the" can be used before "fish" to define the part of speech of "can" and the noun of "fish" respectively, such as "They will can the fish." or "They canned the fish"; You can also add an adverbial after the verb to limit the part of speech of the auxiliary verb of "can" and the part of verb of "fish", such as: "They can fish in the pond."

\subsection{Pragmatic Ambiguity}

The above types of ambiguity are caused by language structure. But sometimes ambiguity can be eliminated in proper context. Therefore, in spite of the form of structure, language inevitably has a very important level, which is pragmatic.

Charles H. Morris first defined pragmatics with the intention of distinguishing pragmatics from syntax and semantics in his work Foundations of the Theory of signs. As Geoffrey Leech stated in 1974, pragmatics is the study of how utterances have different meanings in different situations [6]. On account of this, people usually relate pragmatics to ambiguity. Pragmatics deals with specific utterances in specific contexts, with particular emphasis on the ways in which different social contexts influence language interpretation. In other words, pragmatics is concerned with the way language is used to communicate, not the way it is composed. It puts the language structure in a certain context for research, that is, a word or sentence has different meanings in different contexts. In the process of language application, as the context changes, a language form will show multiple meanings, which is called pragmatic ambiguity.

Thomas describes the process of interpreting as several levels under the heading of pragmatics: The first level is that of abstract meaning; we move from abstract meaning to contextual meaning (also called utterance meaning) by assigning meaning and/or reference to a word, phrase or sentence. The third level of meaning is reached when we consider the speaker's intention, known as the force of an utterance. Ambiguity might appear at each level of the interpreting process [7]. 
E.g. 5: Mr. Smith telephoned his father that he did not know the truth.

Without any hint, the pronoun "he" refers to unknown, or "Mr. Smith" or "his father", then this sentence can only be translated as:

a. Mr. Smith called his father and said that he is not himself Understand the truth.

b. Mr. Smith called his father and said that his father did not understand the truth.

\subsection{Syntactic Ambiguity}

From the syntactic point of view, Lyons gives a definition that, any sentence (according to generative grammar) which can make more than one parsing is ambiguous [8]. Syntactic ambiguity means that the structure of a sentence can be analyzed differently, that is, the components of the sentence have different ways of combinations, which results in ambiguity. Therefore, syntactic ambiguity is also called structural ambiguity. Compared with other types of ambiguity, syntactic ambiguity appears more frequently in ordinary English, and is more complicated than other types of ambiguity.

The ambiguity that arises because people have different understandings of the syntactic structure in a sentence is called syntactic ambiguity. At the syntactic level, the study of ambiguity is mainly based on the theoretical basis of transformational generative grammar. According to the transformational generative grammar theory, each sentence has a surface structure and a deep structure. The surface structure refers to the relationship between the various components of the sentence that is actually formed, and the linear arrangement of these components results in the sentence. The deep structure describes the grammatical relationships that are the basis of the surface structure, and these grammatical relationships cannot be directly seen from the surface structure. Therefore, if a surface structure has more than two sentences with deep structures at the same time, ambiguity occurs. Hirst proposed a kind of "attachment theory" by observing a large number of ambiguous sentences. His theory believes that ambiguity is caused by the uncertain attachment relationship between a certain component and other components in the surface structure [9].

\subsubsection{Ambiguity Caused by Adjective Phrases}

E.g. 6: There stood a big brick house at the foot of the hill.

"Big" is an adjective and is used as an attributive in a sentence. It can be regarded as modifying the noun phrase "brick house", or it can be regarded as directly modifying "house". Therefore, this sentence is ambiguous.

\subsubsection{Ambiguity Caused by Adverbial Phrases}

E.g. 7: The General Manager promised a bonus for each employee later.

There is a time adverbial "later" in the sentence. Because it is placed at the end of the sentence, the sentence is ambiguous. This sentence can be translated as "the general manager promises to the bonus will be later given every employee", 
and it can also be translated as "Later the general manager promises to give every employee a bonus".

\subsubsection{Ambiguity Caused by Comparative Sentences}

In English, comparative adverbial clauses often take the form of omission, which can easily lead to ambiguity.

E.g. 8: I love Mary than Lucy.

This sentence is an ambiguous sentence. There are two explanations. The first is "I love Mary better than Lucy does. (=than Lucy loves Mary)"; the second is "I love Mary better than I love Lucy.” (I love Mary more than loving Lucy).

\subsubsection{Ambiguity Caused by the Same Verb Forms}

English verbs have three basic inflection forms, namely the past tense, the past participle and the present participle. The past tense and past participle of most verbs are formed by adding -ed after the original form of the verb, and the present participle is formed by adding -ing after the original form of the verb, and gerunds are also formed by adding -ing after the original form of the verb. Some current participles are converted into adjectives, but still retain the original spelling form; some past participles are also retained as the original spelling form when they are used as adjectives. The variations of the above verbs and their syntactic function conversion often cause ambiguity.

E.g. 9: Flying planes can be dangerous.

"Flying" can be interpreted as a gerund and present participle. When "flying" is the present participle, it is used as a form word to modify the nouns "planes" and "flying". When used as a gerund, it is used in conjunction with "planes" to form a verb phrase and act as a subject in the sentence. Therefore, there are two translations of this sentence: "An airplane in flight may be dangerous" and "Flying an airplane may be dangerous.”

\subsubsection{Ambiguity Caused by Parallel Parts}

Two parallel sentence components are usually connected by parallel conjunctions, such as and. Sometimes there is a modifier before or after the parallel structure, which can easily cause ambiguity.

E.g. 10: Mr. Stone was a professor and a dramatist of great fame.

The syntactic relationship between the parallel structure "a professor and a dramatist" and the attributive "of great fame" makes this sentence ambiguous. Because the attributive "of great fame" can be understood as modifying only "dramatist", but also as modifying "dramatist" and "professor" at the same time.

\subsubsection{Ambiguity Caused by the Unclear Negative Center}

The negative form of English sentences is often composed of "auxiliary verb + negative word (not/n't)". This kind of negative sentence can negate a specific component in the sentence, or it can negate the whole sentence. In negative sentences, "negative scope" and "negative center" are two worthy research objects. The so-called negative range is the main item of negative judgment. The nega- 
tion center is the bearer of the negation meaning. Different understandings of the scope of negation or the center of negation often lead to ambiguity.

E.g. 11: I don't teach because it is easy.

Looking at this sentence in isolation, the following two explanations are acceptable:

a. Because English is easy, I don't want to teach English.

b. I teach English not because English is easy.

\section{Conclusions}

In the previous parts, a variety of issues concerned with ambiguity have been studied. It is really difficult to give a satisfactory and comprehensive explanation that a sentence has two or more unrelated meanings due to phonetic, lexical, pragmatic and syntactic indeterminacy.

In order to help people to get a clearer understanding, this paper reviews the previous analyses and discussions on ambiguity. As a phenomenon of language, ambiguity must have its structural causes of generation, which is more essential than other factors.

This paper focuses on exploring the syntactic analysis of ambiguity in English sentence, citing some English examples as illustration. Yet this paper does not explain in detail the semantic and pragmatic aspects of ambiguity sentences which would be the more practical usage of the language. At the same time, ambiguity is not only the different meanings we need to avoid or eliminate, on the other hand, ambiguity is a useful and powerful device which we can use in humors, advertisements, literature works and so on.

In short, when encountering such ambiguous sentences, in addition to analyzing the specific context, we should also make logical judgments. To make correct logical judgments, we must have a wide range of knowledge other than language. All in all, ambiguity is a more complicated issue, which deserves to be taken seriously. Analyzing ambiguity can help us better understand and use language.

\section{Funding}

This article is funded by the special fund project of the basic scientific research business expenses of the central universities of Southwest Minzu University in 2020, project number: 2020SYB10.

\section{Conflicts of Interest}

The author declares no conflicts of interest.

\section{References}

[1] Jacobs, R.A. and Rosenbaum, P.S. (1968) English Transformational Grammar. Blaisdell Publishing Co., Waltham.

[2] Kempson, R.M. (1979) Ambiguity and Word Meaning. Studies in English Linguis- 
tics. Longman Group Ltd., London.

[3] Wu, Q.G. (1988) Introduction to Semantics. Hunan Education Press, Changsha.

[4] Carroll, L. (1865) Alice's Adventures in Wonderland. Random House, New York.

[5] Lewis, C. (1871) Through the Looking Glass. Random House, New York.

[6] Leech, G.N. (1983) Principles of Pragmatics. Longman, London.

[7] Thomas, J. (1995) Meaning in Interaction: An Introduction to Pragmatics. Longman, London.

[8] Lyons, J. (1977) Semantics. Cambridge University Press, Cambridge.

[9] Hirst, G. (1987) Semantic Interpretation and the Resolution of Ambiguity. Cambridge University Press, Cambridge. https://doi.org/10.1017/CBO9780511554346 